Revista científica, arbitrada e indizada, bajo la modalidad electrónica.

Recibido: $22 / 02 / 2020$

Aceptado: 19/03/2020

\title{
LA GESTIÓN DEL CONOCIMIENTO: UN ESLABÓN FORMADO POR UN DÚO; COMPLEJIDAD Y ENTORNO ORGANIZACIONAL
}

Knowledge Management: A link formed by a duo; complexity and organizational environment

\author{
Alber Molina ${ }^{1}$ \\ Petróleos de Venezuela, S.A (PDVSA) \\ molina.alber@gmail.com \\ Venezuela
}

\section{RESUMEN}

La gestión del conocimiento constituye un aspecto en la cultura del cambio que permite mejorar continuamente el nivel de competencias, logrando un crecimiento organizacional para alcanzar nuevas oportunidades y proyectos. El presente artículo tiene como propósito establecer la vinculación de la gestión del conocimiento con la complejidad y el entorno organizacional, como estrategia para el cierre de brechas y adquisición de competencias requeridas. Fue abordado a través de un enfoque cualitativo, desde una perspectiva interpretativa, a través del método hermenéutico (teórico-documental). En este sentido, se concluye que las empresas inmersas en un entorno organizacional cambiante y complejo, característico de la dinámica y actual situación de Venezuela, han acelerado la fuga de talento altamente calificado, perdiendo conocimientos y capacidades innovadoras para alcanzar posiciones competitivas, por lo que la gestión del conocimiento permite la transferencia, generación y adquisición de nuevos conocimientos y capacidades innovativas para el efectivo cumplimiento de metas y objetivos estratégicos.

Palabras clave: Gestión del conocimiento, competencias gerenciales, entorno organizacional, complejidad.

\section{ABSTRACT}

Knowledge management is an aspect in the culture of change that allows continually improving the level of skills, achieving organizational growth to reach new opportunities

\footnotetext{
${ }^{1}$ Geoquímico. Magister Scientiarum en Ciencias Geológicas. Doctorando en Ciencias Gerenciales. Director Ejecutivo de Formación y Capacitación de PDVSA.
} 


\section{Revista científica, arbitrada e indizada, bajo la modalidad electrónica.}

and projects. The purpose of this article is to establish the relationship of the knowledge management with complexity and the organizational environment, as a strategy for closing gaps and acquiring the required skills. It was approached through a qualitative approach, from an interpretive perspective, through the hermeneutical method (theoreticaldocumentary). It is concluded that companies immersed in a changing and complex organizational environment, characteristic of the dynamic and current situation of Venezuela, have accelerated the flight of highly qualified talent, losing knowledge and innovative capacities to reach competitive positions, which is why knowledge management allows the transfer, generation and acquisition of new knowledge and innovative capacities for the effective fulfillment of strategic goals and objectives.

Key words: Knowledge management, management skills, organizational environment, complexity

\section{INTRODUCCIÓN}

Las empresas han comenzado a darse cuenta de la importancia de saber qué es lo que saben y de hacer el mejor uso de este conocimiento (Macintosh, 1997). El conocimiento representa el activo más importante y el recurso económico significativo de las empresas, por lo cual constantemente se definen estrategias para adquirirlo, administrarlo, además de retenerlo (Drucker, 1995).

La gestión del conocimiento es un proceso que permite transferir los conocimientos o experiencias dentro de una organización, facilitando la transferencia de información y habilidades de manera sistemática y eficiente, estableciendo objetivos, estrategias, coherencia, integración y contextualización para investigar, crear, compartir, conectar, acceder, socializar, intercambiar, adaptar, aplicar e innovar conocimiento.

A su vez, permite la dinamización de la cultura organizacional para el mejoramiento académico - profesional, transformando la capacidad de conocimiento, aprendizaje, investigación e innovación. Implica ir mucho más allá de un simple plan de formación, por lo que es esencial para favorecer la estructura corporativa, a fin de lograr mejoras en la competitividad del entorno organizacional, el cual se ha visto afectado por los contantes cambios del mundo globalizado, aspectos dinámicos y situación actual del país, lo que lo convierten en un entorno gerencial de alta complejidad, donde debe incorporarse adecuadamente el manejo de conocimientos, creatividad, desarrollo e innovación de enfoques proactivos. 


\section{Revista científica, arbitrada e indizada, bajo la modalidad electrónica.}

En los últimos años, plenos albores del siglo XXI y debido a la situación actual, las empresas han evidenciado un incremento acelerado en la fuga de talento altamente preparado en áreas gerenciales y especializadas, perdiéndose el conocimiento que durante años se ha podido adquirir, para lo cual se han invertido altas sumas de dinero, razón por la cual se hace necesario e indispensable el manejo del conocimiento e información como aspectos determinantes tanto para la excelencia, como para la competitividad en las empresas y la calidad en la toma de decisiones asertivas y oportunas, permitiendo resolver las situaciones de complejidad que se presentan constantemente tanto en el entorno organizacional actual de Venezuela como del resto del mundo.

La gestión del conocimiento e información ha estado en constante complejidad debido a la explosión de la oferta y la demanda de información, así como al desarrollo de tecnologías de información y comunicación durante el siglo XXI, por lo que deben existir cambios organizacionales de manera de involucrar el conocimiento en cada uno de sus miembros, abarcando e involucrando las sociedades generadoras y gestoras de conocimientos (Torres y Lamenta, 2015).

Estas exigencias requieren que las empresas realicen innovaciones en procesos organizacionalmente complejos, así como desarrollo e implementación de estrategias y capacidades de aprendizaje, gestionando el conocimiento para satisfacer las verdaderas necesidades, avances científicos - tecnológicos, trascendiendo positivamente hacia una adecuada competitividad, oportuna y acertada toma de decisiones, cumplimiento de metas y objetivos estratégicos. A tal efecto, es importante que las empresas analicen e identifiquen el conocimiento disponible requerido; planifiquen y controlen las acciones para generar activos de conocimientos que permitan alcanzar las competencias necesarias para el cumplimiento de proyectos organizacionales.

Por esta razón, el propósito de este artículo consiste en establecer la vinculación de la gestión del conocimiento con la complejidad y el entorno organizacional, como estrategia para el cierre de brechas, apuntando a la adquisición de competencias requeridas para la efectiva ejecución de funciones así como oportuna y acertada toma de decisiones, 


\section{Revista científica, arbitrada e indizada, bajo la modalidad electrónica.}

para lo cual fue aplicado un enfoque cualitativo, desde una perspectiva interpretativa, haciendo uso del método hermenéutico en el contexto teórico - documental.

El desarrollo del presente artículo contempla el resumen, introducción, la descripción metodológica aplicada en la investigación, así como los distintos puntos de interés y atención que fueron abordados teórico, documental e interpretativamente para dar cumplimiento al objetivo propuesto, y así plasmar los aportes y consideraciones finales.

\section{LA GESTIÓN DEL CONOCIMIENTO EN LAS ORGANIZACIONES: UNA COMUNIDAD INTRA E INTERORGANIZACIONAL.}

El conocimiento organizacional es generado en el interior de una creciente comunidad de interacción, que atraviesa niveles y fronteras intra e interorganizacionales, el cual amplifica el conocimiento creado por los individuos y los solidifica como parte de una red de conocimientos e información de la organización (Nonaka y Takeuchi, 1999). De acuerdo a este enfoque, las potenciales fuentes de conocimiento (individuos, grupos, equipos, proyectos, áreas, departamentos, entre otros), respaldan e inciden directamente en la creación y generación de conocimiento.

El conocimiento se considera como la unidad analítica básica necesaria para explicar el comportamiento de las empresas. La organización no solo lo procesa, sino que además lo crea. Para ello, debe considerarse que el valor de la organización ya no reside sólo en sus bienes tangibles, sino en los conocimientos especializados de su personal, experiencia y propiedad intelectual.

Se vislumbra una sociedad del conocimiento, donde el mundo empresarial y de los negocios será cada vez más cercano a las exigencias de nuevas formas del saber, derivado de las experiencias, investigaciones y formas útiles para la sociedad, y así resolver presuntas problemáticas. En este sentido, el espíritu empresarial, no puede reducirse simplemente a la expresión de voluntad, decisión, capacidad de asumir riesgos o cualquier otra manifestación que implique emotividad, sino requiere de conocimientos e investigación para poder inclinarse por un mundo empresarial exitoso. 


\section{Revista científica, arbitrada e indizada, bajo la modalidad electrónica.}

Tanto el conocimiento como la investigación son fuentes de la creación y desarrollo empresarial, donde la dinámica actual, entorno organizacional y complejidad, hacen que se requiera de una gran dosis de ingenio y creatividad para visualizar los cambios y necesidades demandados actualmente. Por ello, las empresas deben adaptarse, ajustarse y anticiparse a los cambios, proyectando adecuadamente sus estrategias a los aportes de su capital intelectual con el propósito de alcanzar metas y objetivos organizacionales (Villarroel, 2017).

Se requiere de una gestión basada en competencias para lograr que dichos procesos resulten eficientes, así como capacidad para desarrollar eficazmente un trabajo, utilizando conocimientos, habilidades y destrezas como atributos necesarios que faciliten solucionar situaciones complejas y problemas.

Resulta de gran importancia fomentar en las empresas el aprendizaje organizacional, con la finalidad de buscar y mantener la excelencia, niveles de competitividad, adoptando cambios en la filosofía de acción, donde las personas representan el principal valuarte de las empresas, el más importante y de mayor valor agregado en los procesos.

La verdadera ventaja competitiva de una organización radica en el conocimiento existente para identificar, resolver y afrontar nuevas situaciones y problemas, por lo que el conocimiento representa un recurso altamente valioso, y su capacidad de aprendizaje es la acción más estratégica que se debe desarrollar. El aprendizaje dinámico a nivel individual y organizacional, depende de la habilidad, disposición y herramientas que tengan las organizaciones para adquirir, generar y transformar el conocimiento en prácticas más adecuadas, permitiendo adaptarse y respondiendo efectivamente a las condiciones del entorno (Villarroel, 2017).

Según Mai (2004), el aprendizaje organizacional es un proceso en el cual las organizaciones adquieren y generan conocimiento a través de sus trabajadores, con el objetivo de convertirlos en generación de saber institucional que le permita adaptarse a las condiciones de su entorno, y así establecer espacios de aprendizaje desde el individuo en la organización y de la organización al individuo. En otras palabras, está asociado al trabajo organizacional que se realiza activamente con la finalidad de mejorar prácticas y así buscar 


\section{Revista científica, arbitrada e indizada, bajo la modalidad electrónica.}

nuevas y mejores formas de hacer las cosas. Son organizaciones que se autoevalúan en un entorno en constante cambio.

La mayoría de las investigaciones en cuanto al aprendizaje en las organizaciones coinciden en que este tipo de aprendizaje se presenta a través de los individuos en primera instancia y después por medio de la socialización en comunidades de trabajo y de aprendizaje.

Cada organización presenta una situación particular, y la diferencia entre ellas y su capacidad de aprender se relaciona con aspectos como la influencia del entorno y en cómo interactúan cada uno de sus componentes, la acción diferenciada de los gerentes como líderes, la forma de organización, historia de la institución, las expectativas con relación a la mejora, y la complejidad (Fernández, 2011).

El ser competitivo es una cualidad organizacional que guarda estrecha relación con su personal, ya que si una organización es competitiva será porque su personal también lo es. La calidad de la empresa es consecuencia de su talento humano, por lo que el continuo mejoramiento y desarrollo de los trabajadores no es una exigencia por cumplir, sino un requisito para el éxito del negocio. Toda organización debe diseñar, desarrollar e implementar estrategias de transferencia y gestión del conocimiento que se produce en la empresa, creando competencias esenciales para la ejecución de funciones, desarrollo de planes - proyectos, cumplimiento de metas y objetivos estratégicos.

Carballo (2006), considera la gestión del conocimiento como el conjunto de prácticas, herramientas, técnicas y metodologías que permiten identificar cuáles son los conocimientos más adecuados para llevar a cabo una determinada actividad y desarrollar sus planes futuros, y así garantizar la disponibilidad de estos conocimientos, protegerlos y utilizarlos eficientemente.

En consecuencia, la gestión del conocimiento se refiere al cambio en los principios de las personas, donde existe un tránsito de la información que se posee, compartiendo conocimientos que benefician a toda la organización, transformando la información, 


\section{Revista científica, arbitrada e indizada, bajo la modalidad electrónica.}

conocimiento y capital intelectual en un valor duradero para la organización, lo que genera ideas e información de alto valor e impacto para la empresa y sus respectivos procesos.

Durante el siglo XXI, uno de los desafíos consiste en establecer cómo se aprende en el marco del trabajo de las organizaciones. La formación y gestión del conocimiento adquieren, desde la perspectiva del cambio, entorno organizacional y complejidad, un valioso valor estratégico en la sociedad del conocimiento (Maragall, 2002).

Aprender será necesario para vivir, convivir y en última instancia sobrevivir en términos productivos, saber qué conocimiento y donde está, será cada vez más importante en el éxito de las organizaciones; resulta fundamental manejar de manera más eficiente y eficaz el conocimiento para poder adaptarse al entorno e innovar en los niveles regionales, nacionales e internacionales, por lo que se debe iniciar un proceso de cambio donde el principal objetivo sea establecer soluciones radicales que apunten a la competitividad y sostenimiento de las organizaciones, impactando favorablemente en el comportamiento de las mismas e influenciando en la sociedad, estableciendo una cultura frente a la gestión documental y del conocimiento, coadyuvando y fortaleciendo todos los componentes de una corporación o empresa.

Dibella y Nevis (1998) han señalado la diferencia que marca el proceso de aprendizaje en las organizaciones, donde los actores se involucren activamente en las iniciativas de aprendizaje, así como en mantener un clima propicio para aprender. En consecuencia, desde este punto de vista sostengo que se hace necesario e importante comprender e interpretar cómo el entorno organizacional y la complejidad, emergen constantemente en las nuevas concepciones de la realidad, lo cual plantea la necesidad de indagar cómo ejecutan sus acciones para el aprendizaje organizacional y cómo se genera el conocimiento.

Los planteamientos señalados destacan la presencia de nuevas formas de organizaciones; donde la dinámica, los constantes cambios y la situación actual, generan entornos organizacionales cada vez más complejos, enfocando acciones que permitan cumplir con los retos y desafíos que se presentan continuamente producto de los contantes cambios del mundo globalizado. 


\section{Revista científica, arbitrada e indizada, bajo la modalidad electrónica.}

\section{ENTORNO ORGANIZACIONAL Y COMPLEJIDAD FUNDAMENTADA EN CINCO PRINCIPIOS: PLANIFICAR, ORGANIZAR, DIRIGIR, COORDINAR Y CONTROLAR.}

El estudio de las organizaciones demanda superar los fundamentos administrativos que subyacen al mundo organizacional del siglo $\mathrm{XX}$, donde se aprecian los aportes de la teoría clásica de Taylor, Fayol y Max Weber. Estas teorías postularon el diseño de organizaciones bajo la perspectiva de un pensamiento mecanicista y burocrático, sustentado en un modelo de trabajo definido y organizado de forma jerárquica (Castillo, 2013). La Teoría Clásica de Frederick Taylor "Padre de la Administración Científica", explica cómo el elevar la productividad en una organización se logra al tener personal específicamente seleccionado, con una buena remuneración y en un buen ambiente de trabajo; al que se pueda capacitar y desarrollar para trabajar en conjunto, buscando los mejores resultados.

La Teoría Administrativa de Henry Fayol "Padre de la Administración Moderna" se fundamenta en cinco elementos que forman el proceso administrativo: planificar, organizar, dirigir, coordinar y controlar. Por su parte, el sociólogo Max Weber, a principios de siglo $\mathrm{XX}$, dio al término burocracia en las organizaciones formales modernas el carácter de poseer una estructura racional y especializada de las distintas funciones y actividades con orientación hacia objetivos explícitos y precisos. La influencia de estas corrientes y concepciones persisten en el transcurrir del tiempo; y aún en la plenitud del presente siglo se evidencian organizaciones que se basan en la división y especialización de las tareas, con estructuras jerárquicas, piramidales y funcionales.

Wheartley (1997), plantea una nueva manera de comprender las organizaciones, desde la percepción de un universo en constante cambio, dentro de una red de patrones conectivos, en donde éste se reordena por medio de procesos creativos y dinámicos e incorpora constantes cambios que mantienen un orden y apertura en los sistemas, con capacidad de renovarse a sí mismo permitiendo la existencia de fuerzas invisibles que estructuran el espacio y mantienen unidas las cosas complejas.

A partir de estos planteamientos, Monasterio (2013), sostiene que las perspectivas del entorno organizacional y de la complejidad representan una epistemología para 


\section{Revista científica, arbitrada e indizada, bajo la modalidad electrónica.}

adentrarse en el conocimiento de las organizaciones, más aún en donde se han agotado sus lógicas para responder a organizaciones embebidas dentro de contextos complejos caracterizados como sistemas abiertos, dinámicos, en interacciones constantes con un entorno repleto de conflictos y turbulencias.

González (2011), argumenta que las organizaciones deben ser abordadas como sistemas abiertos, dinámicos, con capacidad de formar redes en constante innovación. Realidad en donde sus actores no forman parte de un mecanismo cerrado, sino que se motivan en un camino abierto con el rumbo de una visión compartida.

De acuerdo a ello, la visión de las organizaciones debe estar constituida con base a las acciones con las cuales se gestionan, con planteamientos que inviten e incentiven a una nueva forma de pensar y abordar la ejecución de los distintos procesos y actividades, considerando cabalmente los constantes y dinámicos cambios que se presentan en el entorno organizacional y que sin lugar a duda generan escenarios de alta complejidad.

Asimismo, Wheartley (1997) establece que las organizaciones emergentes experimentan cambios profundos caracterizados por la complejidad. Además, la revolución de la tecnología de la información y de las telecomunicaciones, impone nuevos modos de interacción, donde son visualizadas organizaciones como sistemas vivos, enérgicamente abiertos, impregnadas de un pensamiento sistémico.

Es así como las organizaciones deben ser transformadas en sistemas abiertos y dinámicos, con capacidad de mantener la constante innovación, generar conocimiento e información, estableciendo mecanismos que permitan su administración exitosa y oportuna, donde los actores involucrados se incentiven a lograr un camino abierto con visión compartida, considerando el conocimiento humano y científico como ejes fundamentales para el replanteamiento del entorno organizacional.

El contexto mundial impone desafíos y retos que estiman la emergencia de necesidades encaminadas hacia la información, conocimiento, administración del conocimiento y la manera en que se interpreta la realidad. En este sentido, la sociedad demanda profundos y complejos cambios organizacionales como producto de 


\section{Revista científica, arbitrada e indizada, bajo la modalidad electrónica.}

circunstancias histórico - social que pueden enmarcarse dentro de los cuatro tipos de organizaciones descritos por Katz y Kahn (1989).

Por consiguiente, los citados autores, establecen las organizaciones productivas o económicas, dedicadas a proporcionar bienes y servicios. Seguido por las organizaciones dedicadas a la socialización y el adiestramiento para los papeles que ocuparán en otras organizaciones y en la sociedad como un todo, a las cuales los autores las denominan organizaciones de mantenimiento. Organizaciones adaptativas, las cuales buscan crear nuevos conocimientos y dar soluciones a las presuntas problemáticas y/o situaciones. Finalmente, las organizaciones político-administrativas, que coordinan a la gente y los recursos materiales, físicos, entre otros.

En este trayecto surgen nuevos enfoques para el estudio de las organizaciones, como plantea Monasterio (2013), es una necesidad imperante superar el pensamiento lineal, ante la necesaria combinación de posibilidades que persiguen una visión mucho más real de los sistemas humanos, propia del devenir irreversible y la estabilidad, como factores de evolución del sistema social. Se deben orientar los procesos desde una perspectiva compleja con comprensión social y humana, donde lo emocional, lo ético, lo moral, cultural, el aprendizaje, el conocimiento e información, son categorías que sin lugar a duda deben estar consideradas.

En el mismo orden de ideas, la propuesta de Senge (2012), acerca de las organizaciones que aprenden, considera abandonar la idea de un mundo compuesto por fuerzas separadas, desconectadas y crea el término de organizaciones inteligentes, donde la gente expande continuamente su aptitud para generar los resultados deseados, cultivan nuevos y expansivos valores de pensamiento, quedando en libertad la aspiración colectiva, donde las personas continuamente aprenden a aprender en conjunto.

Una organización aprende cuando mantiene una filosofía enfocada hacia la anticipación, reacción, respuesta al cambio, complejidad, incertidumbre, haciendo hincapié en que la única fuente sustentable de ventajas competitivas es la tasa de crecimiento de sus conocimientos, así como la manera en que se gestione y transfiera (Senge, 2012). 


\section{Revista científica, arbitrada e indizada, bajo la modalidad electrónica.}

Igualmente, la Teoría de Acción, propuesta por Argyris y Schön (1989), enfatiza el comportamiento y el aprendizaje humano en el contexto de las organizaciones. Dichos autores han estudiado sistemáticamente el problema de la relación pensamiento - acción desde el punto de vista de una teoría que concibe al hombre como un ser que diseña sus acciones, las ejecuta y evalúa sus consecuencias, responden a sus intenciones y las modifica o trata de modificarlos, cuando los resultados le son adversos.

Según Picón (1994), en la Teoría de Acción se entiende la conducta como algo constituido por los significados y por las intenciones de los actores. Así, estos diseñan acciones mediante las cuales tratan de lograr las consecuencias que están en sus intenciones, y controlan esas acciones para saber si han resultado efectivas; dan sentido al ambiente en el cual se desenvuelven construyendo significados que se constituyen en guías para sus acciones.

De acuerdo a ello, la acción y el aprendizaje humano, en relación directa con el entorno organizacional y la complejidad, se ubican en el contexto más amplio del conocimiento, donde estudiar el comportamiento de las organizaciones implica una teoría de acción para dar sentido a las funciones, planes, proyectos, y de esta manera lograr el cumplimiento exitoso de los objetivos planteados.

\section{METODOLOGÍA}

El abordaje de la investigación se realizó a través de un enfoque cualitativo, apoyado en un supuesto epistemológico, referido a la forma de relación que se da entre el investigador y lo investigado, para establecer los criterios a través de los cuales se determina la validez del conocimiento. Fue utilizado el método hermenéutico en el contexto teórico - documental, desde una perspectiva interpretativa, y de esta manera describir, comprender e interpretar las ideas y prácticas que permiten construir la realidad estudiada.

\section{ANÁLISIS INTERPRETATIVO}

Como resultado de esta investigación teórica - documental, desde una perspectiva interpretativa, pudo evidenciarse que a pesar de los distintos períodos de tiempo contemplados en la cronología del entorno organizacional, la complejidad y la gestión del 


\section{Revista científica, arbitrada e indizada, bajo la modalidad electrónica.}

conocimiento, siendo este último el más reciente y moderno en relación a las dos teorías mencionadas (complejidad y entorno organizacional), todos en conjunto guardan estrecha relación con los contantes y dinámicos cambios, producto del mundo globalizado, generando entornos organizacionales con alta complejidad, limitando o dificultando los procesos que se ejecutan a través de patrones tradicionales.

Por lo que es necesario implementar estrategias modernas en continuo desarrollo, investigación e innovación, para lograr la competitividad de las organizaciones, y así posicionar los productos, bienes y servicios, rivalizando en el contexto regional, nacional e internacional, y sobrepasando las barreras u obstáculos que generan el constante dinamismo organizacional y complejidad.

La gestión del conocimiento coadyuva en la adquisición y fortalecimiento de competencias gerenciales y especializadas o técnicas, para la adecuada ejecución de planes $\mathrm{y}$ proyectos, oportuna toma de decisiones y exitoso cumplimiento de metas y objetivos estratégicos, desarrollando una cultura basada en el conocimiento compartido, donde el equipo gerencial posee las competencias necesarias para incentivar el conocimiento y el talento de sus colaboradores mediante la motivación.

Los integrantes de una organización son los pilares fundamentales para el aprendizaje organizacional, quienes actúan y toman decisiones de acuerdo a las estrategias establecidas, lo cual favorece a que los individuos actúen de forma organizada para el cumplimiento de planes y objetivos organizacionales.

Siguiendo el razonamiento anterior, sea como individuos o como organizaciones, se necesita de una base de conocimientos que sean capaces de transformarse o renovarse a sí mismos cuando sea necesario. Tales conocimientos han de ser significativos y útiles en la práctica del contexto al que se apliquen, y actuarán como semilla para la generación de conocimiento. En consecuencia, transitar hacia el aprendizaje organizacional implica codificar el intelecto para desarrollar racionalmente la inteligencia, y de esta manera no solo crear conocimiento sino también gestionarlo, tomando importancia la transferencia del conocimiento como un aspecto de la administración del mismo. 


\section{Revista científica, arbitrada e indizada, bajo la modalidad electrónica.}

Es importante destacar, que las empresas inmersas en esquemas organizacionalmente complejos desarrollan una constante evolución, innovación y aprendizajes continuos, con la finalidad de alcanzar ventajas competitivas sostenibles en el tiempo, proyectando el mayor éxito empresarial, de manera que podrá crear y desarrollar su capacidad de innovación mediante una adecuada gestión de sus recursos y capacidades.

El aprendizaje organizacional permite el desarrollo de nuevos conocimientos y se considera como un requisito para el desarrollo tecnológico, convirtiéndose en el mejor camino para utilizar los recursos de la organización, y más aún en el contexto cambiante y de alta complejidad que se presenta en la actualidad, permitiendo afrontar los cambios y desarrollando nuevos conocimientos en los trabajadores, quien a su vez representan los activos intangibles más importante de la organización, con disposición, creatividad y habilidades para la realización de planes y proyectos.

El entorno empresarial actual público y privado, se encuentra inmerso en un escenario de alta complejidad producto de la situación actual del país, cuyos esquemas organizacionales requieren cambiar los enfoques tradicionales, orientándolos a la competitividad, eficiencia, eficacia y efectividad que permitan generar soluciones inmediatas, por lo que se deben diseñar, desarrollar e implementar estrategias alternativas, dentro de las cuales se encuentra la gestión del conocimiento como un eslabón primordial para el cierre de brechas, adquisición de competencias requeridas, ejecución de planes proyectos y cumplimiento de metas y objetivos estratégicos en entornos organizacionales complejos.

\section{CONSIDERACIONES FINALES}

Las teorías sobre el aprendizaje y la creación de conocimiento en las organizaciones, constituyen el modo en que estas transforman sus visiones, igual conocimientos individuales en capacidad organizacional y conocimientos colectivos.

Es así como la formación y gestión del conocimiento adquieren, desde la perspectiva del cambio, un importante valor estratégico en la sociedad del conocimiento, de la mano al desarrollo del siglo XXI. No obstante, la gestión del conocimiento, sin lugar a duda 


\section{Revista científica, arbitrada e indizada, bajo la modalidad electrónica.}

coadyuva en la adquisición y fortalecimiento de competencia para la adecuada ejecución de proyectos, oportuna toma de decisiones, así como exitoso cumplimiento de metas y objetivos estratégicos establecidos.

Asimismo, el aprendizaje permanente se encuentra directamente asociado a organizaciones que poseen alta capacidad para tomar decisiones innovadoras, haciendo uso mínimo de tiempo, información, conocimiento, recursos financieros y talento humano.

De acuerdo a ello, como un todo fundamentado a través de entornos organizacionales complejos, resulta necesario saber cuál es y dónde está el conocimiento y así coadyuvar al éxito de las organizaciones.

Resulta fundamental manejar de manera eficaz y eficiente el conocimiento para poder adaptarse al entorno e innovar en los niveles regionales, nacionales e internacionales.

El mundo globalizado genera entornos organizacionales con alta complejidad que limitan o dificultan la ejecución de procesos a través de patrones tradicionales, por lo que la gestión del conocimiento en estrecha relación con la teoría del entorno organizacional y la teoría de la complejidad, permite afrontar los desafíos y retos producto de la dinámica actual.

Los constantes cambios organizaciones requieren nuevos esquemas de gestión, donde la comunicación, información, conocimiento y liderazgo se conviertan en aspectos estratégicos de los negocios, dando respuestas oportunas en el contexto empresarial actual. En este sentido, los contantes cambios económicos, políticos, sociales, culturales y tecnológicos que orientan la dinámica actual del estado venezolano, hacen necesario diseñar, desarrollar e implementar prácticas, métodos, estrategias y procedimientos que permitan trascender en la cultura organizacional y su desempeño.

\section{REFERENCIAS BIBLIOGRÁFICAS}

Argyris, C., Schön, D. (1989). Participatory Action Research and Action Science Compared: A commentary. American Behavioral Scientist. 32(5), 612.623.

Carballo, R. (2006). Innovación y gestión del conocimiento. Madrid: editorial Díaz de Santos.

Castillo, V. (2013). Teoría de las Organizaciones. México: Trillas. 


\section{Revista científica, arbitrada e indizada, bajo la modalidad electrónica.}

Dibella, A., Nevis, E. (1998). How Organizations Learn. San Francisco, EE. UU: JosseyBass Publishers.

Drucker, P. (1995). The information executives truly need. Hardvard Buseness Review.

Fernández, C. (2011). Modelo sobre competencias gerenciales para el personal directivo de tecnología del sector financiero basado en enfoque de organizaciones inteligentes. Tesis Doctoral - Tecana American University. 138 p.

González, M. (2011). Los cambios en las organizaciones. Consultado en: http://www.eumed.net/ce/2011a/mgt.htm

Katz, D., Kahn, R. (1989). Psicología social de las organizaciones. México: Trillas.

Macintosh, A. (1997). Position paper on knowledge-creating company: How Japanese company create the dinamics of innovation. Oxford University Press.

Mai, R. (2004). Leadership for School improvement: Cues for organizational learnig and renewal efforts. [versión electrónica], The Educational Forum. (68)3, 211-221.

Maragall, E. (2002). Las comunidades de práctica como experiencia formativa para la mejora de las administraciones públicas.(en línea) En: VII Congreso Internacional del CLAD sobre la Reforma del estado y de la Administración Pública (08/11/2002), Lisboa, Portugal: Centro Latinoamericano de Administración para el Desarrollo CLAD.

Monasterio, D. (2013). La última frontera de la gerencia. Aportes para la construcción de una teoría desde el pensamiento emergente. Editado por Monasterio Dilia. Caracas.

Nonaka, I., Takeuchi, H. (1999).La Organización Creadora de Conocimiento. México: Oxford University Press.

Picón, G. (1994). El proceso de Convertirse en Universidad. Aprendizaje Organizacional en la Universidad Venezolana. Caracas: Fondo Editorial de la Universidad Pedagógica Experimental Libertador.

Senge, P. (2012). La quinta disciplina. Como impulsar el Aprendizaje en la Organización Inteligente. México: Ediciones Granica, S.A.

Torres, K., Lamenta, P. (2015). La gestión del conocimiento y los sistemas de información en las organizaciones. Revista Científica Electrónica de Ciencias Gerenciales. 11(32), 3-20.

Villarroel, X. (2017). Aprendizaje organizacional en el sistema nacional de orquestas venezolano. Tesis Doctoral - Universidad Nacional Experimental de la Fuerza Armada Nacional Bolivariana (UNEFA). 167 p.

Wheartley, M. (1997). El liderazgo y la nueva ciencia. Barcelona: Granica. 